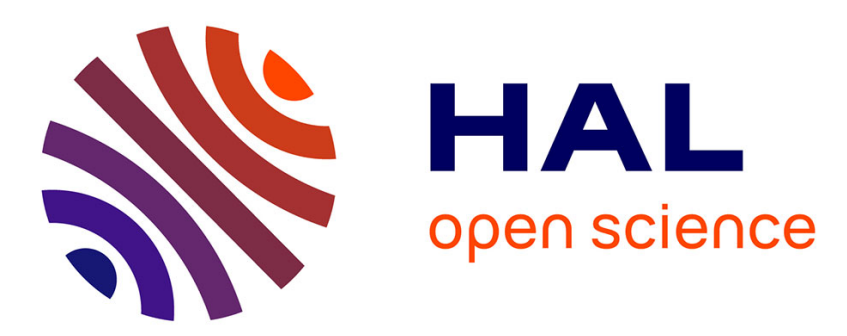

\title{
CARACTÉRISATION DE COUCHES MINCES DE VERRES DE CHALCOGENURE PRÉPARÉES PAR PECVD
}

B. Cros, Henri Camon, Y. Brocheton, J. Gonchond, A. Tissier, J. Balladore, M. Ribes

\section{To cite this version:}

B. Cros, Henri Camon, Y. Brocheton, J. Gonchond, A. Tissier, et al.. CARACTÉRISATION DE COUCHES MINCES DE VERRES DE CHALCOGENURE PRÉPARÉES PAR PECVD. Journal de Physique Colloques, 1989, 50 (C5), pp.C5-343-C5-351. 10.1051/jphyscol:1989541 。 jpa-00229564

\section{HAL Id: jpa-00229564 https://hal.science/jpa-00229564}

Submitted on 1 Jan 1989

HAL is a multi-disciplinary open access archive for the deposit and dissemination of scientific research documents, whether they are published or not. The documents may come from teaching and research institutions in France or abroad, or from public or private research centers.
L'archive ouverte pluridisciplinaire HAL, est destinée au dépôt et à la diffusion de documents scientifiques de niveau recherche, publiés ou non, émanant des établissements d'enseignement et de recherche français ou étrangers, des laboratoires publics ou privés. 


\title{
CARACTERISATION DE COUCHES MINCES DE VERRES DE CHALCOGENURE PREPAREES PAR PECVD
}

B. CROS, H. CAMON*, Y. BROCHETON**, J.P. GONCHOND**, A. TISSIER* * J.L. BALLADORE* et M. RIBES

Laboratoire de Physicochimie des Matériaux Solides, UA-407, Université des Sciences et Techniques du Languedoc, Place Eugène-Bataillon, F-34060 Montpellier Cedex 1, France

- Laboratoire d'optique Electronique du CNRS, 29, rue Jeanne Marvig. F-31055 Toulouse, France

* "CNET-Grenoble, BP. 98, chemin du Vieux-Chêne, F-38243 Meylan, France

\begin{abstract}
Résumé - Des couches minces de verres de chalcogénure, de compositions $\overline{\mathrm{GeSe}}_{3}$ et GeSe $\mathrm{G.5}_{5.5}$, déposées par PECVD sont caractérisés par differentes techniques de microscopie électronique. La structure hétérogène mise en évidence sur le matériau de composition GeSe ${ }_{5.5}$ parất également

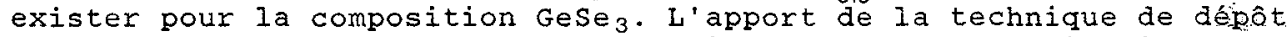
par PECVD pour préparer des couches minces en vue d'applications. microlithographiques est discuté.
\end{abstract}

\begin{abstract}
Thin films of chalcogenide glasses, with GeSe 3 and GeSe 5.5 compositions, were prepared by PECVD. They were characterized by various techniques of electronic microscopy. The heterogeneous. structure, which appears at the GeSe 5.5 composition, seems also to exist for the GeSe 3 composition. The influence of the PECVD method on the microlithographic applications of this films is discussed.
\end{abstract}

\section{1 - INTRODUCTION}

Les verres de chalcogénures, et plus particulièrement lés verres GeSey. ont fait l'objet de nombreux travaux au cours des dix dernières annés en raison de leurs applications comme matériaux photosensibles. Aprés sensibilisation à I'argent, ces matériaux sont utilisables comme résines photosensibles et se caractérisent en microlithographie par :

- un contrasté trés élevé:

- une bonne sensibilité dans un large domaine spectral (UV, UV lointain, rayons $\mathrm{x}$ et électrons):

- une sélectivité élevée, vis à vis des plasmas de gravure, entre les régions insolées et les régions non insolées (développement à sec);

- une bonne résistance au plasma d'oxygène utilisé pour la gravure de la couche inférieure de polymère;

- une résolution submicronique.

De nombreuses techniques sont utilisées pour obtenir le matériau sous forme de couches minces : pulvérisation cathodique /1/, tournette /2/, évaporation sous vide /3/, pulvérisation magnétron /4/ et, plus récemment, procédé CVD assisté plasma $/ 5 /$.

\section{2 - METHODES EXPERTMENTALES}

2.1 - Réalisation des couches minces

Les films de verres de chalcogénure Gesey sont déposés dans un réacteur à plasma, à parois chaudes, décrit antérieurement /5,6/. Sa géométrie axiale a été choisie pour faciliter l'application industrielle du procedé. Les substrats de silicium sont placés sur des électrodes verticales de graphite situées dans la zone de temperature homogène du réacteur. Ces électrodes sont reliées à un générateur RF de $400 \mathrm{kHz}$, dont la puissance est réglée à $45 \mathrm{~W}$. 
Les gaz réactifs sont le germane et I'hydrogène sélénié (Alphagaz, qualité N50). Ils sont mélangés à l'azote (Alphagaz, qualité N60) avant l'injection dans le réacteur. Les débits de germane, hydrogène sélénié et azote sont respectivement de 20,50 et $400 \mathrm{~cm}^{3} / \mathrm{mn}$ (contrôleurs de débit massique Tylan) pour obtenir la composition $\mathrm{GeSe}_{3}$ et 11,50 , et $400 \mathrm{~cm}^{3} / \mathrm{min}$ pour $1 \mathrm{a}$ composition GeSe 5,5 : La pression est régulée à $0,87 \mathrm{hPa}$ par une vanne soufflet. Les dépôts, effectués à $100^{\circ} \mathrm{C}$, sont suivis d'un recuit in situ sous azote à la même temperature pendant 1 heure. L'épaisseur des films est mesuree par ellipsométrie (Applied Materials) et Alphastep (Tencor). La composition est contrôlée par microsonde camebax. La qualité de surface des couches est observée par microscopie électronique dे balayage.

\section{2 - Microscopie électronique à balayage (MEB)}

Le microscope électronique à balayage Cámbridge utilisé est de type stereoscan 100. Sa tension d'accélération peut varier de 2 à $25 \mathrm{kV} ;$ son grandissement théorique est de 200000.

Les échantillons ont préalablement subi une attaque chimique par un solvant sélectif. Les solutions de TMAH (hydroxyde de tétraméthylammonium), de concentration $0.1 \mathrm{M}$, sont couramment utilisés en microlithographie. Dans un système biphasé Ge-Se, elles dissolvent préférentiellement lá phase riche en germanium.

\section{3 - Microscopie électronique à transmission (MET)}

Des films des deux compositions GeSe 3 et GeSe 5,5 , d'épaisseur 50 à $70 \mathrm{~nm}$, sont déposés par PECVD sur des substrats de silicium recouverts de polymère PMMA (polymétacrylate de méthyle). Les films sont décollés du substrat dans un bain d'acétone et recueiliis sur des grilles de cuivre de $3 \mathrm{~mm}$ (400 mesh). Les observations sont effectuees avec un microscope Jeol $200 \mathrm{cx}$ à $200 \mathrm{keV}$. Pour obtenir les diagrammes de diffraction et les images, le condenseur est suffisamment défocalisé pour que la densité d'électrons arrivant sur I'objet soit suffisamment faible pour ne pas induire des'modifications rapides. Les épaisseurs de quelques dizaines de $\mathrm{nm}$ ont été choisies afin d'éviter des effets de chauffage et de charge en cours d'observation.

En mode image, deux methodes sont utilisables, champ clair et champ sombre. En mode champ clair, l'image se forme lorsqu'on réalise l'interférence des faisceaux transmis par l'objet sans déviation et des faisceaux diffusés ou diffractés. Pour que l'image soit une reproduction fidèle de l'objet, il faut que ces faisceaux interfèrent en phase, ce qui n'est pas le cas des faisceaux diffractés soumis à différentes aberrations. Avec le diaphragme objectif, tous les faisceaux diffractés ou trop fortement diffusés peuvent être éliminés de la formation de l'image pour en augmenter le contraste. Ca contraste dépend alors de la nature, de l'épaisseur et de la structure de l'objet observé. Dans le cas d'un objet amorphe, il s'agit essentieliement d'un contraste d'épaisseur.

En mode image champ sombre, on sélectionne pour former l'image un faisceau diffracté caractéristique d'une périodicité et on centre le diaphragme sur lui (éliminant ainsi le faisceau transmis sans déviation). Cette technique est notamment utilisée pour visualiser la répartition de la périodicité choisie dans l'objet lorsque 1 'intensité diffractée est suffisante. On obtient alors une image brillante sur fond noir qui indique la localisation des zones diffractantes, c'est-a-dire caractéristiques de la périodicité choisie.

\section{4 - Microanalyses X par MET}

Les prélèvements d'échantillons dans un bain d'acétone sont également recueillis sur une grille de cuivre à maille fine. L'appareil utilisé est un Tracor EDS 422. Les conditions d'analyse sont les suivantes: $120 \mathrm{kV}, \mathrm{G}=$ 8000. L'acquisition des diagrammes $x$ se fait entre 0 et $20 \mathrm{kV} / 10 \mathrm{eV} / \mathrm{ch}, 300$ sec). La focalisation par rapport à l'épaisseur de la couche est schématisée sur la figure 1.

\section{3 - RESULTATS}

3.1 - Microscopie électronique à balayage Les compositions GeSe 3 et GeSe 5,5 ont été choisies pour cette étude de façon a encadrer la composition GeSe 4 pour laquelle, d'aprés plusieurs auteurs, apparait une démixtion $/ 7,8 /$. 

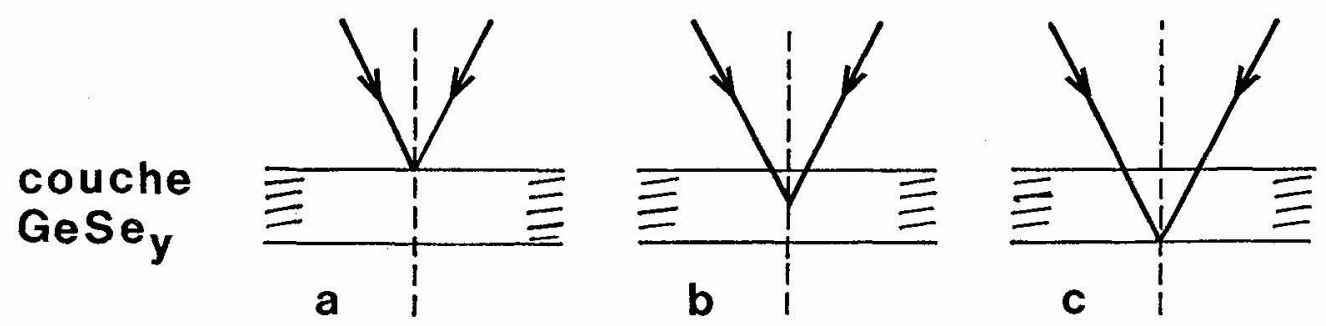

Fig. 1 - Conditions de focalisation. a - Sous-focalisation (franges blanches sur les bords d'un trou). b - Focalisation. c - Sur-focalisation (frange noire sur les bords d'un trou).

a

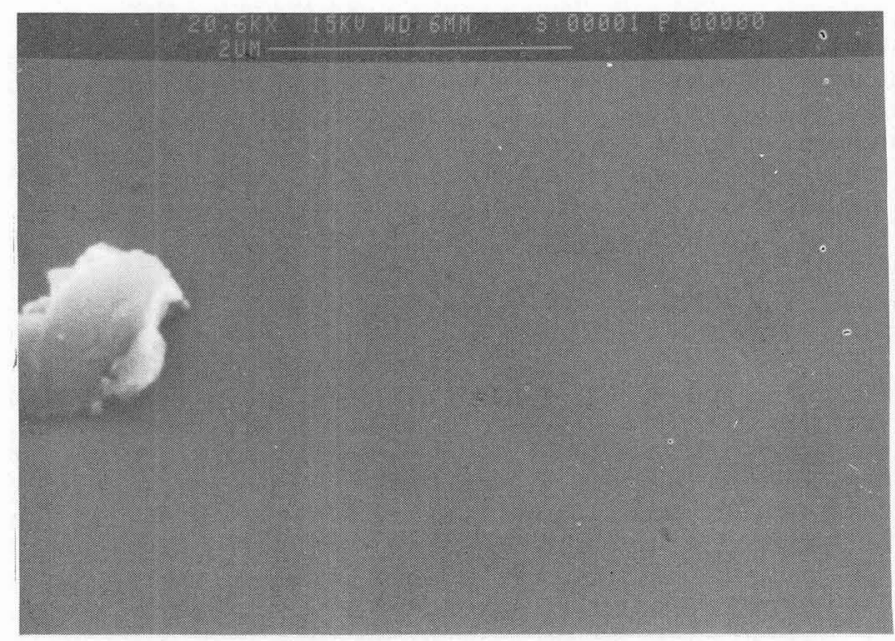

b

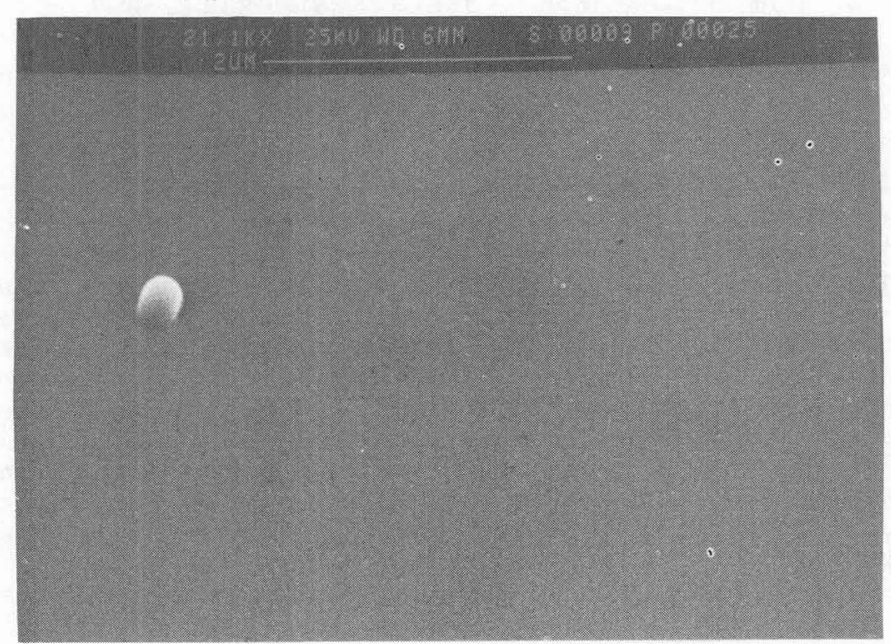

Fig. 2 - Couches de verre GeSey préparées par PECVD avant attaque (a/ $\mathrm{y}=3$. b/ $y=5,5)$. 
En I'absence d'attaque chimique, la surface des couches minces déposées par PECVD ne présente pas de relief (fig. 2). La présence d'un défaut est nécessaire pour effectuer la mise au point. Aprés une attaque chimique (TMAH) de quelques dizaines de secondes, la couche de composition Gese 5.5 laisse apparâtre un relief (attaque différenciée), alors que le matériau de composition GeSe $\mathrm{Ge}_{3}$ garde un aspect homogène (fig. $4 \mathrm{a}$ et $3 \mathrm{a}$ ). Ce phénomène s'accentue avec le temps d'attaques (fig. 4b et $4 \mathrm{c}$ ). Les ilôts, d'une taille moyenne de $100 \mathrm{~nm}$, sont caractéristiques d'une dissolution sélective. L'échantilion de composition $\mathrm{GeSe}_{3}$ ne montre après plusieurs minutes d'attaque chimique qu'une surface légérement granuleuse (fig. $3 a$ et $3 b$ ), la taille des grains restant très petite, de l'ordre de $50 \mathrm{~nm}$. Donc, si pour la composition GeSe 5,5 il semble bien exister deux phases, rien ne permet pour 1 'instant de trancher dans un sens ou dans l'autre pour $\mathrm{GeSe}_{3}$.

3.2 - Microscopie électronique à transmission

En diffraction, des anneaux diffus, caractéristiques d'un matériau amorphe, sont observés. Il est à noter que trois valeurs de périodicité restent identiques, avec les mêmes intensités diffractées, pour les deux compositions étudiées (les distances sont données en nm) :
$\mathrm{GeSe}_{3}$
0,620 faible
0,315 forte
0,180 moyenne
0,117 trés faible

GeSe 5,5

0,578 faible

0,315 forte

0,176 moyenne

0,118 trés faible

En mode de type champ sombre, l'image ne montre pas de contraste localisé, quelle que soit la partie de chacun des anneaux choisie. On peut donc considérer qu'il existe une répartition homogène des périodicités dans 1'objet au grandissement choisi. Ceci dénote la présence d'une seule phase ordonnée et reste compatible avec la coexistence d'une deuxième phase ne possédant pas d'ordre à moyenne distance.

En mode image de type champ clair, des différences de contraste sont visibles. Pour la composition Gese 5,5 , elles confirment l'existence des microdomaines observés par MEB, notamment leur taille de 1 'ordre de $250 \mathrm{~nm}$ (fig. 5b). La composition Gese 3 montre des contrastes analogues dénotant cependant des microdomaines nettement plus petits, de 1 ordre de $20 \mathrm{~nm}$ (fig. 5 a).

\section{3 - Microanalyses X par MET}

La couche de GeSe 3 apparalt homogene, les analyses confirmant la composition. L'observation de la couche de GeSe 5,5 révele des grains sombres de 200 à 300 $\mathrm{nm}$ de dimension moyenne et des grains clairs toujours plus petits (\# $100 \mathrm{~nm}$ en moyenne). La focalisation successive sur chaque type de grain confirme leur différence de composition. Une série d'analyses de la composition des grains sombres donne pour $y$ la valeur moyenne de $8,0+1-1,4(y=6,8-7,15$ $-9,1-7,1-7,8-7,6-8,6-5,6-8,4-8,7-11,0)$. Une série analogue d'analyses de la composition des grains clairs donne une valeur moyenne de $5,3+/-0,3(y=5,9-5,2-5,6-5,1-5,25-4,95-5,05-5,31)$.

\section{4 - DISCUSSION}

La seule référence utilisable pour l'etude structurale des verres Gesey est le composé cristallisé GeSe $\mathrm{G}_{2}$. Sa structure est constituée de tétraèdres Gese 4 partageant un sommet ou une arête selon leur position dans les chânes formant les feuillets /9/. Dans les travaux récents, la structure du verre GeSe ${ }_{2}$ est décrite grâce au modele des "clusters", ceux-ci étant considérés comme des fragments de la structure cristalline /10-15/. Ce type de structure désignée sous le nom de "outrigger raft" comporte principalement des tétraèdres GeSe 4 déformés reliés par les arêtes ou les sommets. La différence essentielle avec la structure de GeSe 2 cristallisé est l'existence de liaisons se-se terminant dans certaines directions les chaines de tétraèdres. Ces liaisons seraient compensées par la formation de liaisons Ge-Ge dans des petites entités de type éthane. Mais, si la présence de liaisons se-se semble bien établie, a notre connaissance, aucun auteur ne précise la distribution des entités de type éthane à l'intérieur de la matrice vitreuse. La taille des clusters a eté estimée à $5 \mathrm{~nm}$ environ, ce qui correspond à six chânes de tétraères joints par les sommets /11/. Selon Phillips/16,17/, la formation de verres de composition Gesey telle que y soit supérieur à 2 résulte de 1 'addition dans le réseau d'atomes de sélénium : les tétraèdres ayant pour sommet commun un atome de selénium deviennent alors reliés par une liaison se-se. Pour ces compositions $(y>2)$ existent donc deux types de liaisons se- 
C5-347

a
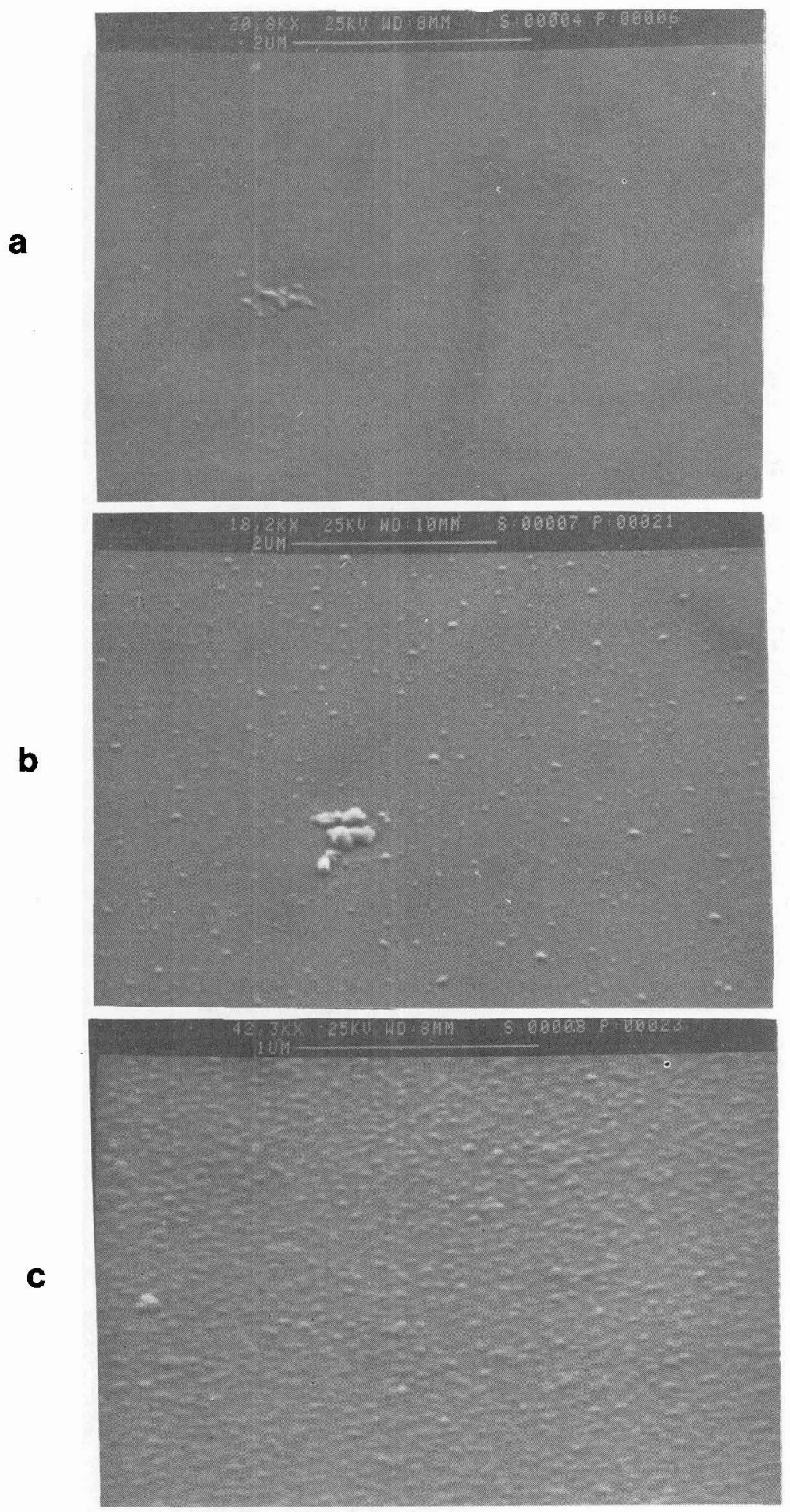

Fig. 3 - Couche de composition $\mathrm{GeSe}_{3}$ aprés une attaque sélective (solution de TMAH 0,1 M) de 20 secondes (a), 2 minutes (b) et 5 minutes (c). 


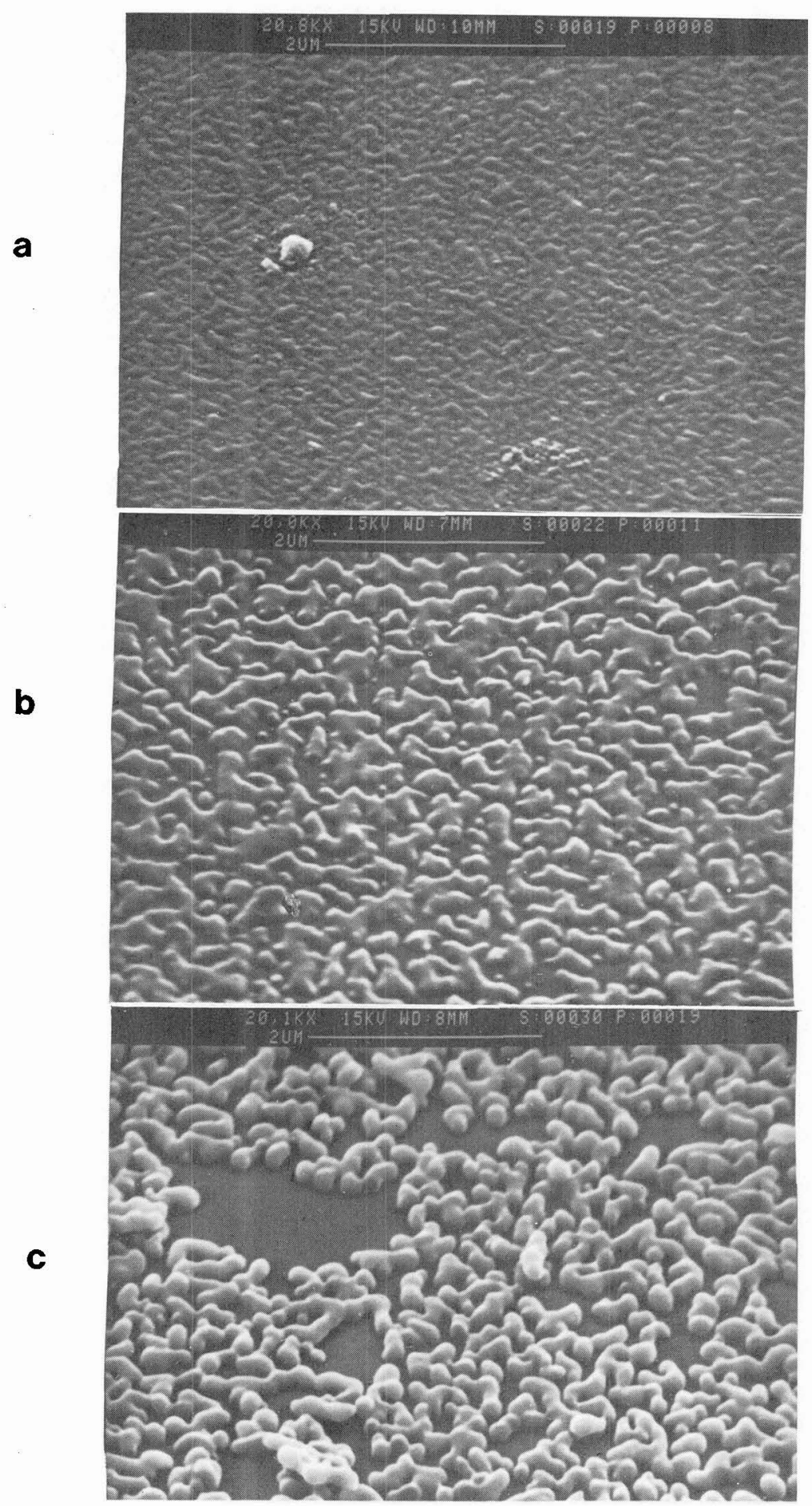

Fig. 4 - Couche de composition GeSe $_{5,5}$ aprés une attaque sélective (solution de TMAH $0,1 \mathrm{M}$ ) de 20 secondes (a), 2 minutes (b) et 5 minutes (c). 


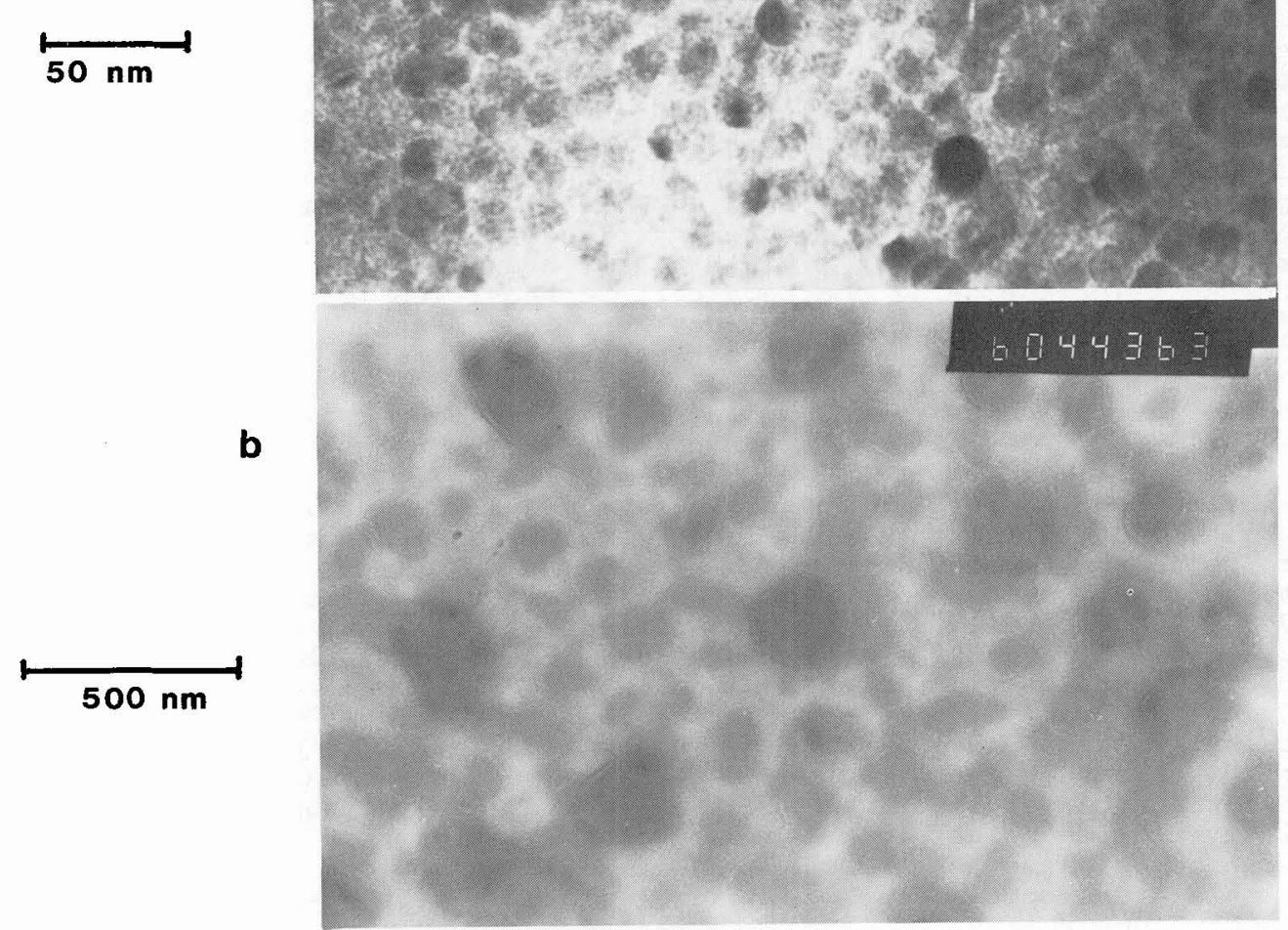

Fig. 5 - Image de type champ clair en MET d'une couche de verre Gesey déposée par PECVD, $a / y=3$, grossissement 400 000. b/ $y=5,5$, grossissement 60000 .

Se, les unes terminant les chasnes dans une même direction de l'outrigger raft, les autres participant à la chaîne pour relier deux tétraèdres. Le réseau ne peut accueillir qu'un nombre fini d'atomes de sélénium et ce type de structure est limité à la composition GeSe 4 . Les verres plus riches en sélénium $(y>4)$ seraient le siège d'une démixtion, les atomes de sélénium ne pouvant s'insérer dans le réseau s'associant en chaines sen et en amas $\left(\mathrm{Se}_{\mathrm{n}}\right)_{\mathrm{m}}$.

Nous avons confronté cette hypothèse à nos résultats expérimentaux.

4.1 - Microscopie électronique à balayage

L'attaque d'une couche mince de composition GeSe 5.5 laisse subsister au bout de 2 à 3 minutes des "nodules" d'une taille de l'ordre de 200 à $500 \mathrm{~nm}$.

Le réactif aissolvant préférentiellement les phases Gesey riches en germanium, ces nodules peuvent être considérés comme des zones "riches en sélénium". Ce résultat est cohérent avec l'hypothèse de Phillips et les résidus d'attaque seraient alors des agglomérats de sélénium.

L'attaque d'une couche mince de composition GeSe parait beaucoup plus 
homogène, ce qui est logique si on considère qu'il s'agit d'un matériau monophasé, la composition limite GeSe y (y \# 4) n'étant pas atteinte. Aprés 2 a 3 minutes d'attaque apparaissent cependant de petites hétérogénéités sous la forme de nodules de trés petite taille $(10$ a $20 \mathrm{~nm})$. Ces grains restent trés dispersés.

\section{2 - Microscopie électronique à transmission}

Les résultats de la diffraction et les observations en mode image champ sombre ne diffèrent pas notablement de la composition $\mathrm{GeSe}_{3}$ à la composition $\mathrm{GeSe}_{5,5}$. Le phénomène éventuel de démixtion du sélénium au dela de la composition $\mathrm{GeSe}_{4} \mathrm{n}$ 'est pas de nature a apporter des modifications; les atomes de sélenium se rassemblant sous la forme de chaines ou d'amas ne font apparaftre aucun ordre à moyenne distance et donc aucune périodicité nouvelle dans 1 'organisation atomique.

L'apparition en mode image champ clair de zones de contraste différent est la seule traduction en microscopie électronique a transmission des nodules observés par microscopie électronique à balayage aprés attaque sélective de la couche de composition GeSe 5.5 . Les tailles mesurés selon les deux techniques sont peu differentes pour une même composition. L'analogie des observations en mode image champ clair des compositions correspondant d y $=3$ et 5,5 traduit une morphologie semblable. Une structure hétérogene dès la composition $\mathrm{GeSe}_{3}$ n'est donc pas à exclure, la taille des microdomaines éventuels étant très petite (nettement plus peti.te que celle des microdomaines observés pour $\mathrm{y}=5,5)$, de 1 'ordre de quelques dizaines de $\mathrm{nm}$. L'attaque sélective de 1 a couche à cette composition ( $y=3$ ) fait apparaitre des grains de même taille. Si on admet que la demixtion dans les verres massifs apparaft pour y \# 4, Ies caractéristiques de la couche obtenue par PECVD pour $y=5,5$ s'expliquent bien. En revanche, la structure hétérogène observée pour $y=3$ ne peut pas s'expliquer par ce mécanisme.

4.3 - Microanalyse X par MET

Nous avons essaye, par une technique fine d'analyse, de vérifier la composition des différents domaines observés grâce aux techniques de microscopie électronique.

L'analyse des domaines riches en germanium conduit à la composition moyenne de $\mathrm{GeSe}_{5,3}$. Cette valeur de y est supérieure à celle qui est généralement admise comme limite du domaine monophasé, Gese 4 . La différence peut être attribuée à une participation des domaines décrits comme des agglomérats de sélénium entourant le domaine analysé. Ces zones "riches en sélénium" ont, d'après 1'analyse une composition moyenne de GeSe 8,0 . La même raison (participation des zones riches en germanium entourant la zone analysé) peut être donnée pour expliquer ce résultat dans un domaine supposé formé exclusivement de sélénium. Une deuxieme explication peut être avancée : la séparation de phase ne conduirait pas à des compositions limites GeSe ${ }_{4}$ et $\mathrm{Se}_{n}$, mais à des compositions où, dans les deux cas, le germanium serait présent, l'une voisine de $\mathrm{GeSe}_{4}$ et l'autre beaucoup plus riche en sélénium.

\section{5 - CONCLUSION}

Parmi les nombreuses méthodes, antérieures au procédé PECVD, utilisées pour la réalisation de couches minces de verres de séléniure de germanium, la comparaison au plan des applications lithographiques reste difficile en raison du manque de donnees expérimentales. Toutefois, nous pouvons souligner qu:elles possedent une caractéristique commune : le conditionnement en couche mince s'effectue en deux étapes dont la première est la préparation du matériau sous forme massive. Cette étape préalable est réalisée par cofusion. Les films déposés à partir du matériau massif ont des compositions telles que $2<y<9$. Lors de cette deuxième opération, des écarts à la stoechiométrie d'origine sont enregistrés : les couches obtenues par evaporation continue s'enrichissent en sélénium, tandis que les évaporations rapides favorisent un enrichissement en germanium. Dans l'alternative de dépot par tournette, la contamination du film par des espèces hydrocarbonées peut avoir une influence notable sur la sensibilité du chalcogénure au photodopage. La pulvérisation cathodique, plus fréquemment utilisée, nécessite la réalisation de cibles de diamètre important pour atteinare une homogénéité en épaisseur satisfaisante sur de grandes surfaces. Ceci est difficile à pratiquer avec ce type de matériau. De plus, chaque nouvelle 
composition nécessite une cible différente; enfin, si les films, obtenus par cette technique ont une qualité satisfaisante, la vitesse de dépôt est faible, ce qui pourrait être un obstacle au plan industriel.

Pour notre part, nous avons développé au laboratoire une technique de dépôt par réaction chimique en phase vapeur à partir de germane et d'hydrogène sélénié. Les verres de chalcogénure ont des températures de transition vitreuse relativement basses (de 230 à $95{ }^{\circ} \mathrm{C}$ quand $y$ varie de 2 à 9 pour les verres de séléniure de germanium). D'autre part, la technologie bicouche, de même que la nécessité d'éviter des migrations d'éléments d'une couche à l'autre en cours de dépôt pour réalisation de microcomposants imposent une température de réaction peu élevée. Pour répondre à ces impératifs, nous avons opté pour la technique PECVD afin de substituer partiellement l'apport énergétique du plasma à l'apport énergétique d'origine thexmique. Par ailleurs, cette méthode doit favoriser un transfert industriel. En effet, elle permet de traiter simultanément plusieurs dizaines de plaquettes et se prête bien à I'automatisation. Les performances lithographiques du système séléniure d'argent-séléniure de gexmanium sont tributaires des techniques d'obtention des films minces. Nous avons montré que. le verre de chalcogénure déposé par PECVD se distingue par une microstructure hétérogène (biphasée) liée à la méthode de préparation utilisée. Il est donc nécessaire de qualifier ce matériau en microlithographie pour comparer ses caractéristiques à celles des couches obtenues par d'autres techniques. C'est le but d'etudes menées par ailleurs /18/.

\section{REFERENCES}

/1/ YOSHIKAWA, A., OCHI, O., NAGAI, H. et MIZUSHIMA, Y., Appl. Phys. Lett. $31(3)(1977) 161$.

/2/ CHERN, G.C. et LAUKS, I., J. Appl. Phys. 53(10) (1982) 6979.

/3/ CHEN, C.H., J. Non-Crystal. Solids 44 (1981) 391.

/4/ UTSUGI, Y., YOSHIKAWA, A, et KITAYAMA, T., Microelectronic Engineering 2 (1984) 281

/5/ RIBES, M., CROS, B, et JULIEN, P., Optical Microlithographic Technology for Integrated Circuit Fabrication and Inspection, H.L. STOVER et S. WITTECOEK Chairs/Editors, SPIE 811 (1987) 202.

$/ 6 /$ JULIEN, P., GRANNEMAN, E., CROS, B. et RIBES, M., vide Couches Minces 237 suppl. (1987) 37 .

$17 /$ PHILLIPS, J.C.. J. Non-Crystalline Solids 34 (1979) 153.

$18 /$ PHILlIPS, J.C., J. Non-Crystaline Solids $\underline{43}$ (1981) 37 .

/9/ von DITTMAR, G. et SCHAEFER, H, Acta Cryst. B32 (1983) 2726.

/10/ KAWAMOTO, Y. et KAWASHIMA, C., Mat. Res. BulI. 17 (1982) 1511.

/11/ BRIDENBAUGH, P.M., ESPINOSA, G.P., GRIFFITHS, J.E., PHILLIPS, J.C. et REMEIKA, J.P., Phys. Rev. B20 (1979) 4140.

112/ GRIFFITHS, J.E., PHILLIPS, J.C. ESPINOSA, G.P., REMEIKA, J.P. et BRIDENBAUGH, P.M., Phys. Stat. Sol. b122 (1984) K11.

/13/. ARONOVITZ, J.A., BANAVAR, J.R., MARCUS, M.A. et PHILLIPS, J.C., PhYs. Rev. B28 (1983) 4454 .

/14/ GRIFFITHS, J.E., ESPINOSA, G.P., REMEIKA, J.P. et PHILLIPS, J.C., PhYS. Rev. B25 (1982) 1272 .

/15; GRIFFITHS, J.E., ESPINOSA, G.P., PHILLIPS, J.C. et REMEIKA, J.R., PhYS. Rev. B28 (1983) 4444 .

/16/ PHILLIPS, J.C., Phys. Rev. B31 (1985) 8157.

$117 /$ PHILLIPS, J.C., PrOC. Symp. Inorganic Resists systems, DOANE et HELLER eds. $82(9)(1982) 147$.

/18/ BROCHETON, Y., Thèse, Montpellier, 1989. 